\title{
Towards a validated model for affective classification of texts
}

\author{
Michel Généreux and Roger Evans \\ Natural Language Technology Group (NLTG) \\ University of Brighton, United Kingdom \\ $\{$ M.Genereux, R.P.Evans\}@brighton.ac.uk
}

\begin{abstract}
In this paper, we present the results of experiments aiming to validate a twodimensional typology of affective states as a suitable basis for affective classification of texts. Using a corpus of English weblog posts, annotated for mood by their authors, we trained support vector machine binary classifiers to distinguish texts on the basis of their affiliation with one region of the space. We then report on experiments which go a step further, using four-class classifiers based on automated scoring of texts for each dimension of the typology. Our results indicate that it is possible to extend the standard binary sentiment analysis (positive/negative) approach to a two dimensional model (positive/negative; active/passive), and provide some evidence to support a more fine-grained classification along these two axes.
\end{abstract}

\section{Introduction}

We are investigating the subjective use of language in text and the automatic classification of texts according to their subjective characteristics, or 'affect'. Our approach is to view affective states (such as 'happy', 'angry') as locations in Osgood's Evaluation-Activation (EA) space (Osgood et al. , 1957), and draws on work in psychology which has a long history of work seeking to construct a typology of such affective states (Scherer, 1984). A similar approach has been used more recently to describe emotional states that are expressed in speech (Cowie and Cornelius, 2002; Schröder and Cowie, 2005). Our overall aim is to determine the extent to which such a typology can be validated and applied to the task of text classification using automatic methods. In this paper we describe some initial experiments aimed at validating a basic two dimensional classification of weblog data, first with Support Vector Machine (SVM) binary classifiers, then with Pointwise Mutual Information - Information Retrieval (PMI-IR). The domain of weblog posts is particularly well-suited for this task given its highly subjective nature and the availability of data, including data which has been author-annotated for 'mood', which is a reasonable approximation of 'affect'.

Recent attempts to classify weblog posts have shown modest, but consistent improvements over a 50\% baseline, only slightly worse than human performance (Mishne, 2005). One important milestone is the elaboration of a typology of affective states. To devise such a typology, our starting point is Figure 1, which is based on a model of emotion as a multicomponent process (Scherer, 1984). In this model, the distribution of the affective states is the result of analysing similarity judgments by humans for 235 emotion terms ${ }^{1}$ using cluster-analysis and multidimensional scaling techniques to map out the structure as a twodimensional space. The positioning of words is not so much controversial as fuzzy; an affective state such as 'angry' to describe facial expression in speech may have a slightly different location than an 'angry' weblog post. In this model, the well-studied 'sentiment' classification is simply a specific case (left vs. right halves of the space). The experiments we describe here seek to go beyond this basic distinction. They involve an additional dimension of affect, the activity dimension, allowing textual data to be classified into four categories corresponding to each of the four quad-

\footnotetext{
${ }^{1}$ Reduced to less than 100 in Figure 1.
} 


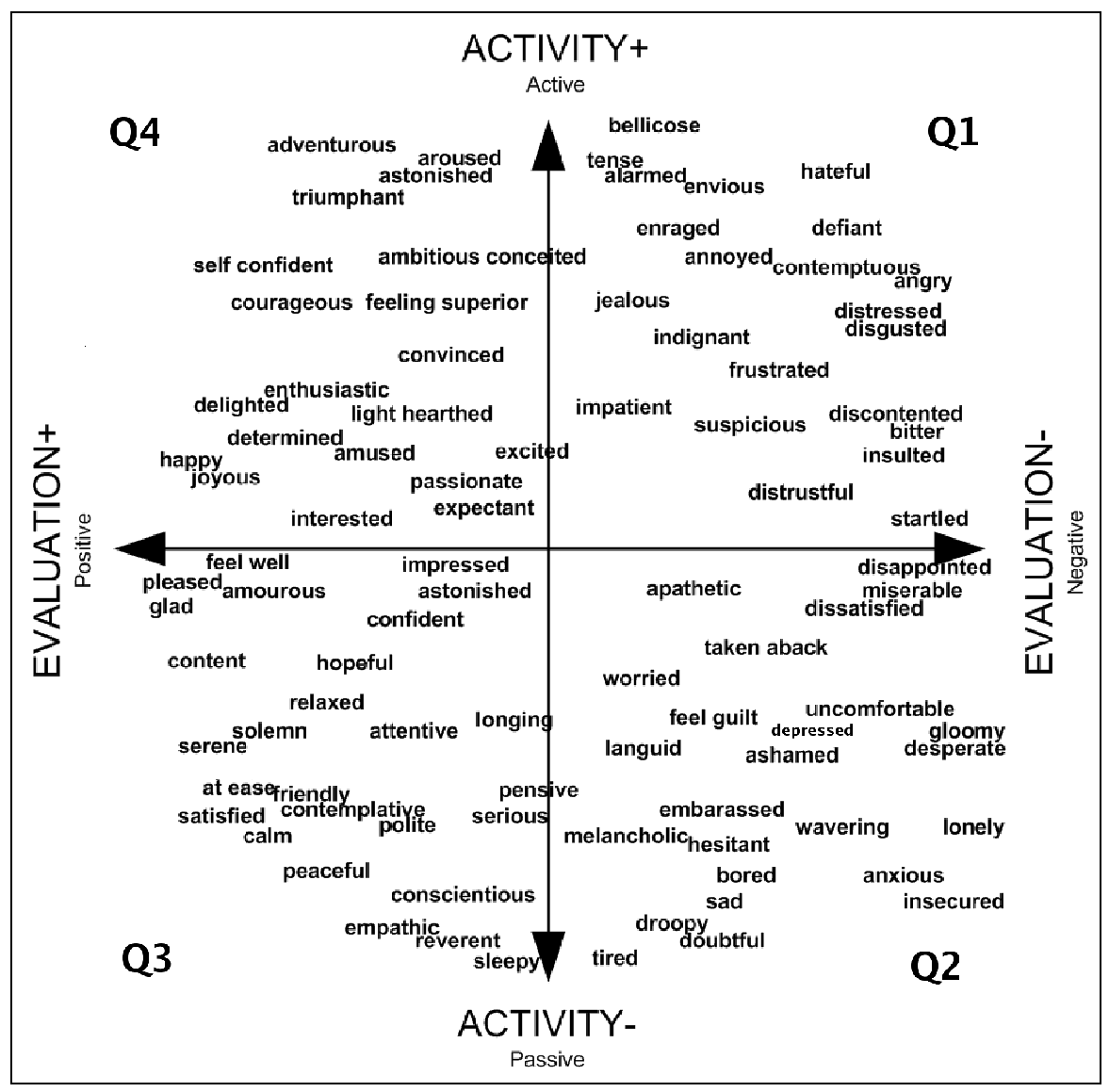

Figure 1: Typology of affective states based on (Scherer, 1984)

rants in the space. Ultimately, once scores have been 'promoted' to real measures, classification can be more precise; for example, a text is not only negative and passive, it is more precisely 'depressive'. With such a more precise classification one might, for example, be able to detect individuals at risk of suicide. In Experiment 1, we use binary classifiers to investigate how the four quadrants defined by the typology hold together, the assumption being that if the typology is correct, the classifiers should perform substantially better than a random baseline. In Experiment 2, we go a step closer towards a more fine-grained classification by evaluating the performance of an unsupervised automated technique for scoring texts on both axes. Both these experiments are preliminary - our long term goal is to be able to validate the whole typology in terms of computationally effective classification.

\section{Corpus}

We have collected from Livejournal ${ }^{2}$ a total of 346723 weblogs (mood-annotated by authors) in

\footnotetext{
${ }^{2}$ http: //www. livejournal.com.
}

English, from which almost half are annotated with a mood belonging to one of the four quadrants, described as follows:

Quadrant1 bellicose, tense, alarmed, envious, hateful, angry, enraged, defiant, annoyed, jealous, indignant, frustrated, distressed, disgusted, suspicious, discontented, bitter, insulted, distrustful, startled, contemptuous and impatient.

Quadrant2 apathetic, disappointed, miserable, dissatisfied, taken aback, worried, languid, feel guilt, ashamed, gloomy, sad, uncomfortable, embarrassed, melancholic, depress, desperate, hesitant, bored, wavering, droopy, tired, insecured, anxious, lonely and doubtful.

Quadrant3 feel well, impressed, pleased, amourous, astonished, glad, content, hopeful, solemn, attentive, longing, relaxed, serious, serene, content, at ease, friendly, satisfied, calm, contemplative, polite, pensive, peaceful, conscientious, empathic, reverent and sleepy.

Quadrant4 happy, ambitious, amused, adventurous, aroused, astonished, triumphant, excited, 
conceited, self confident, courageous, feeling superior, enthusiastic, light hearthed, determined, passionate, expectant, interested, joyous and delighted.

In our experiments, we used 15662 from quadrant Q1 (see Figure 1), 54940 from Q2, 49779 from Q3 and 35634 from Q4.

\section{Experiment 1: Distinguishing the four Quadrants}

Our hypothesis is that the classification of two disjoint sets of moods should yield a classification accuracy significantly above a baseline of $50 \%$. To verify our hypothesis, we conducted a series of experiments using machine learning to classify weblog posts according to their mood, each class corresponding to one particular quadrant. We used Support Vector Machines (Joachims, 2001) with three basic classic features (unigrams, POS and stems) to classify the posts as belonging to one quadrant or one of the three others. For each classification task, we extracted randomly 1000 testing examples, and trained separately with 2000 , 4000, 8000 and 16000 examples. In each case, examples were divided equally among positive and negative examples ${ }^{3}$. The set of features used varied for each of these tasks, they were selected by thresholding each (distinct) training data set, after removing words (unigrams) from the categories poor in affective content (prepositions, determiners, etc.). To qualify as a feature, each unigram, POS or stem had to occur at least three times in the training data. The value of each feature corresponds to its number of occurence in the training examples.

\subsection{Results}

Our hypothesis is that, if the four quadrants depicted in Figure 1 are a suitable arrangement for affective states in the EA space, a classifier should perform significantly better than chance $(50 \%)$. Table 1 shows the results for the binary classification of the quadrants. In this table, the first column identifies the classification task in the form ' $\mathrm{P}$ vs N', where ' $\mathrm{P}$ ' stands for positive examples and ' $\mathrm{N}$ ' for negative examples. The 'Random' row shows results for selecting positive and negative examples randomly from all four quadrants. By

\footnotetext{
${ }^{3}$ For instance,, $1000=500$ positives from one QUADRANT + 500 negatives among the other three QUADRANTS.
}

micro-averaging accuracy for the classification of each quadrant vs all others (rows 10 to 13), we obtain at least $60 \%$ accuracy for the four binary classifications of the quadrants ${ }^{4}$. The first six rows show evidence that each quadrant forms a distinctive whole, as the classifer can easily decide between any two of them.

\begin{tabular}{|c|c|c|c|c|}
\hline \multirow{2}{*}{$\begin{array}{l}\text { Testing } \\
1000 \text { examples }\end{array}$} & \multicolumn{4}{|c|}{ Size of training set } \\
\hline & $2 \mathrm{k}$ & $4 \mathrm{k}$ & $8 \mathrm{k}$ & $16 \mathrm{k}$ \\
\hline Q1 vs Q3 & $67 \%$ & $70 \%$ & $72 \%$ & $73 \%$ \\
\hline Q2 vs Q4 & $61 \%$ & $64 \%$ & $65 \%$ & $67 \%$ \\
\hline Q1 vs & $64 \%$ & $66 \%$ & $68 \%$ & $69 \%$ \\
\hline Q2 vs Q3 & $58 \%$ & $59 \%$ & $59 \%$ & $59 \%$ \\
\hline Q3 vs Q4 & $59 \%$ & $60 \%$ & $60 \%$ & $61 \%$ \\
\hline Q4 vs Q1 & $69 \%$ & $72 \%$ & $73 \%$ & $75 \%$ \\
\hline $\mathrm{Q} 1+4$ vs $\mathrm{Q} 2+3$ & $56 \%$ & $58 \%$ & $58 \%$ & $61 \%$ \\
\hline $\mathrm{Q} 3+4$ vs Q1 & $62 \%$ & $65 \%$ & $67 \%$ & $66 \%$ \\
\hline Random & $49 \%$ & $52 \%$ & $50 \%$ & $50 \%$ \\
\hline$\overline{\mathrm{Q} 1 \text { vs } \mathrm{Q} 2+3+4}$ & $67 \%$ & $72 \%$ & $72 \%$ & $73 \%$ \\
\hline $\mathrm{Q} 2$ vs $\mathrm{Q} 1+3+4$ & $59 \%$ & $60 \%$ & $63 \%$ & $63 \%$ \\
\hline Q3 vs Q1+2+4 & $57 \%$ & $58 \%$ & $58 \%$ & $59 \%$ \\
\hline Q4 vs Q1+2+3 & $60 \%$ & $63 \%$ & $65 \%$ & $65 \%$ \\
\hline Micro-accuracy & $61 \%$ & $64 \%$ & $65 \%$ & $65 \%$ \\
\hline
\end{tabular}

Table 1: Accuracy of binary classification

\subsection{Analysis of Results}

We introduce now table 2 that shows two thresholds of significance (1\% and 5\%) for the interpretation of current and coming results. For example, if we have 1000 trials with each trial having a probability of success of 0.5 , the likelihood of getting at least $53.7 \%$ of the trials right is only $1 \%$. This gives us a baseline to see how significantly well above chance a classifier performs. The SVM algorithm has linearly separated the data for each quadrant according to lexical and POS content (the features). The most sensible explanation is that the features for each class (quadrant) are semantically related, a piece of information which is relevant for the model (see section 4). It is safe to conclude that the results cannot be allocated to chance, that there is something else at work that explains the

${ }^{4}$ Micro-averaged accuracy is defined as:

$$
\frac{\sum_{i}\left(t p_{i}+t n_{i}\right)}{\sum_{i}\left(t p_{i}+t n_{i}+f p_{i}+f n_{i}\right)}
$$

where $t p$ stands for "true positive", $f n$ for "false negative", etc. 


\begin{tabular}{|c|c|c|c|}
\hline Trials & Prob(Success) & $1 \%$ & $5 \%$ \\
\hline \hline 1000 & 0.50 & $53.7 \%$ & $52.6 \%$ \\
\hline 750 & 0.50 & $54.3 \%$ & $53.1 \%$ \\
\hline 500 & 0.50 & $55.2 \%$ & $53.6 \%$ \\
\hline 250 & 0.50 & $57.2 \%$ & $55.2 \%$ \\
\hline \hline 1000 & 0.25 & $28.2 \%$ & $27.3 \%$ \\
\hline 750 & 0.25 & $28.7 \%$ & $27.6 \%$ \\
\hline 500 & 0.25 & $29.6 \%$ & $28.2 \%$ \\
\hline 250 & 0.25 & $31.6 \%$ & $29.6 \%$ \\
\hline
\end{tabular}

Table 2: Statistical Significance

accuracies consistently well above a baseline, and this something else is the typology. These results show that the abstraction offered by the four quadrants in the model seems correct. This is also supported by the observation that the classifier shows no improvements over the baseline if trained over a random selection of examples in the entire space.

\section{Experiment 2: Classification using Semantic Orientation from Association}

Our next goal is to be able to classify a text according to more than four classes (positive/negative, active/passive), by undertaking multi-category classification of texts according to particular regions of the space, (such as 'angry', 'sad', etc.). In order to do that we need a scoring system for each axis. In the following experiments we explore the use of such scores and give some insights into how to transform these scores of affect as measures of affect.

Using binary classifiers, we have already established that if we look at the lexical contents of weblog posts tagged according to their mood by their author, these mood classes tend to cluster according to a two-dimensional typology defined by their semantic orientation: positive or negative (evaluation), active or passive (activity). Beyond academic importance, the typology really becomes of practical interest if we can classify the posts using pre-defined automated scores for both axis. One strategy of scoring is to extract phrases, including single words, which are good indicators of subjectivity in texts, and score them according to how they relate or 'associate' to one or the other extremity of each axis. This strategy, called Semantic Orientation (SO) from Association (A) has been used successfully (Turney and Littman, 2003) to classify texts or adjectives of all sorts according to their sentiments (in our typology this corresponds to the evaluation dimension). According to these scores, a text or adjective can be said to have, for example, a more or less positive or negative evaluation. We will use this strategy to go further in the validation of our model of affective states by scoring also the activity dimension; to our knowledge, this is the first time this strategy is employed to get (text) scores for dimensions other than evaluation. In SO-A, we score the strength of the association between an indicator from the text and a set of positive or negative words (the paradigms Pwords and Nwords) capturing the very positive/active or negative/passive semantic orientation of the axis poles. To get the SO-A of a text, we sum over positive scores for indicators positively related to Pwords and negatively related to Nwords and negative scores for indicators positively related to Nwords and negatively related to Pwords. In mathematical terms, the SO-A of a text is:

$$
\sum_{\text {ind }}^{\text {Text }}\left(\sum_{p}^{\text {Pwords }} A(\text { ind }, p)-\sum_{n}^{\text {Nwords }} A(\text { ind }, n)\right)
$$

where ind stands for indicator. Note that the quantity of Pwords must be equal to Nwords.

To compute A, (Kamps et al. , 2004) focus on the use of lexical relations defined in Word$\mathrm{Net}^{5}$ and define a distance measure between two terms which amounts to the length of the shortest path that connects the two terms. This strategy is interesting because it constrains all values to belong to the $[-1,+1]$ range, but can be applied only to a finite set of indicators and has yet to be tested for the classification of texts. (Turney and Littman, 2003) use Pointwise Mutual Information - Information Retrieval (PMI-IR); PMI-IR operates on a wider variety of multi-words indicators, allowing for contextual information to be taken into account, has been tested extensively on different types of texts, and the scoring system can be potentially normalized between $[-1,+1]$, as we will soon see. PMI (Church and Hanks, 1990) between two phrases is defined as:

$$
\log _{2} \frac{\operatorname{prob}\left(p h_{1} \text { is near } p h_{2}\right)}{\operatorname{prob}\left(p h_{1}\right) * \operatorname{prob}\left(p h_{2}\right)}
$$

PMI is positive when two phrases tend to co-occur and negative when they tend to be in a complementary distribution. PMI-IR refers to the fact

\footnotetext{
${ }^{5}$ http: // wordnet.princeton. edu/.
} 
that, as in Informtion Retrieval (IR), multiple occurrences in the same document count as just one occurrence: according to (Turney and Littman, 2003), this seems to yield a better measure of semantic similarity, providing some resistance to noise. Computing probabilities using hit counts from IR, this yields to a value for PMI-IR of:

$$
\log _{n} \frac{N *\left(\text { hits }\left(p h_{1} N E A R p h_{2}\right)+1 / N\right)}{\left(\text { hits }\left(p h_{1}\right)+1\right) *\left(\text { hits }\left(p h_{2}\right)+1\right)}
$$

where $\mathrm{N}$ is the total number of documents in the corpus. We are going to use this method for computing A in SO-A, which we call SO-PMI-IR. The configuration depicted in the remaining of this section follows mostly (Turney and Littman, 2003).

Smoothing values (1/N and 1$)$ are chosen so that PMI-IR will be zero for words that are not in the corpus, two phrases are considered NEAR if they co-occur within a window of 20 words, and $\log _{2}$ has been replaced by $\log _{n}$, since the natural $\log$ is more common in the literature for log-odds ratio and this makes no difference for the algorithm.

Two crucial aspects of the method are the choice of indicators to be extracted from the text to be classified, as well as the sets of positive and negative words to be used as paradigms for the evaluation and activity dimensions. The five part-ofspeech (POS) patterns from (Turney, 2002) were used for the extraction of indicators, all involving at least one adjective or adverb. POS tags were acquired with TreeTagger (Schmid, 1994) ${ }^{6}$. Ideally, words used as paradigms should be context insensitive, i.e their semantic orientation is either always positive or negative. The adjectives good, nice, excellent, positive, fortunate, correct, superior and bad, nasty, poor, negative, unfortunate, wrong, inferior were used as near pure representations of positive and negative evaluation respectively, while fast, alive, noisy, young and slow, dead, quiet, old as near pure representations of active and passive activity (Summers, 1970).

Departing from (Turney and Littman, 2003), who uses the Alta Vista advanced search with approximately 350 millions web pages, we used the Waterloo corpus ${ }^{7}$, with approximately 46 millions pages. To avoid introducing confusing heuristics, we stick to the configuration described above, but (Turney and Littman, 2003) have experimented with different configuation in computing SO-PMIIR.

\footnotetext{
${ }^{6}$ (Turney and Littman, 2003) uses (Brill, 1994).

${ }^{7}$ http: / / canola1.uwaterloo.ca/.
}

\subsection{The Typology and SO-PMI-IR}

We now use the typology with an automated scoring method for semantic orientation. The results are presented in the form of a Confusion Matrix $(\mathrm{CM})$. In this and the following matrices, the topleft cell indicates the overall accuracy ${ }^{8}$, the POSitive (ACTive) and NEGative (PASsive) columns represent the instances in a predicted class, the $\mathrm{P} / \mathrm{T}$ column (where present) indicates the average number of patterns per text (blog post), E/P indicates the average evaluation score per pattern and $\mathrm{A} / \mathrm{P}$ indicates the average activity score per pattern. Each row represents the instances in an actual class ${ }^{9}$.

First, it is useful to get a clear idea of how the SO-PMI-IR experimental setup we presented compares with (Turney and Littman, 2003) on a human-annotated set of words according to their evaluation dimension: the General Inquirer (GI, (Stone, 1966)) lexicon is made of 3596 words $(1614 \text { positives and } 1982 \text { negatives })^{10}$. Table 3 summarizes the results. (Turney and Littman,

\begin{tabular}{|c|c|c|c|}
\hline (U) 76.4\% & POS & NEG & E/P \\
\hline POS(1614) & $59.3 \%$ & $40.7 \%$ & 1.5 \\
\hline NEG(1982) & $9.6 \%$ & $90.4 \%$ & -4.3 \\
\hline \hline (T) 82.8\% & POS & NEG & E/P \\
\hline POS(1614) & $81.2 \%$ & $18.8 \%$ & 3.2 \\
\hline NEG(1982) & $15.8 \%$ & $84.2 \%$ & -3.6 \\
\hline
\end{tabular}

Table 3: CM for the GI: (U)Us and (T)(Turney and Littman, 2003)

2003) reports an accuracy of $82.8 \%$ while classifying those words, while our experiment yields an accuracy of $76.4 \%$ for the same words. Their results show that their classifier errs very slightly towards the negative pole (as shown by the accuracies of both predicted classes) and has a very balanced distribution of the word scores (as shown by the almost equal but opposite in signs values of E/Ps). This is some evidence that the paradigm words are appropriate as near pure representations of positive and negative evaluation. By contrast,

\footnotetext{
${ }^{8}$ Recall that table 2 gives an interpretation of the statistical signifiance of accuracy, with trials $\approx 750$ and Prob(success) $=0.5$.

${ }^{9}$ For example, in the comparative evaluation shown in table 3 , our classifier classified $59.3 \%$ of the 1614 positive instances as positive and $40.7 \%$ as negative, with an average score of 1.5 per pattern.

${ }^{10}$ Note that all moods in the typology present in the GI have the same polarity for evaluation in both, which is some evidence in favour of the typology.
} 
our classifier appears to be more strongly biased towards the negative pole, probably due to the use of different corpora. This bias ${ }^{11}$ should be kept in mind in the interpretation of the results to come.

The second experiment focuses on the words from the typology. Table 4 shows the results. The

\begin{tabular}{|c|c|c|c|c|}
\hline $\mathbf{8 1 . 1 \%}$ & POS & NEG & P/T & E/P \\
\hline POS(43) & $60.5 \%$ & $39.5 \%$ & 1 & 0.4 \\
\hline NEG(47) & $0.0 \%$ & $100.0 \%$ & 1 & -6.4 \\
\hline \hline $\mathbf{6 6 . 7 \%}$ & ACT & PAS & P/T & A/P \\
\hline ACT(39) & $33.3 \%$ & $66.7 \%$ & 1 & -0.9 \\
\hline PAS(51) & $7.8 \%$ & $92.2 \%$ & 1 & -2.9 \\
\hline
\end{tabular}

Table 4: CM for the Typology affective states

value of 1 under P/T reflects the fact that the experiment amounts, in practical terms, to classifying the annotation of the post (a single word). For the evaluation dimension, there is another shift towards the negative pole of the axis, which suggests that words in the typology are distributed not exactly as shown on figure 1, but instead appear to have a true location shifted towards the negative pole. The activity dimension also appear to have a negative (i.e passive) bias. There are two main possible reasons for that: words in the typology should be shifted towards the passive pole (as in the evaluation case), or the paradigm words for the passive pole are not pure representations of the extremity of the pole ${ }^{12}$.

Having established that our classifier has a negative bias for both axes, we now turn to the classification of the quadrants per se. In the next section, we used SO-PMI-IR to classify 1000 randomnly selected blog posts from our corpus, i.e 250 in each of the four quadrants. Some of these posts were found to have no pattern and were therefore not classified, which means that less than 1000 posts were actually classified in each experiment. We also report on the classification of an important subcategory of these moods called the Big Six emotions.

\footnotetext{
${ }^{11}$ Bias can be introduced by the use of a small corpus, inadequate paradigm words or typology. In practice, a quick fix for neutralizing bias would be to normalize the SO-PMI-IR values by subtracting the average. This work aims at tuning the model to remove bias introduced by unsound paradigm words or typology.

${ }^{12}$ At the time of experimenting, we were not aware of an equivalent of the GI to independently verify our paradigm words for activity, but one reviewer pointed out such a resource, see http://www.wjh.harvard.edu/ inquirer/spreadsheet_guide.htm.
}

\subsection{Results}

Of the 1000 blog posts, there were 938 with at least one pattern. Table 5 shows the accuracy for the classification of these posts.

\begin{tabular}{|c|c|c|c|c|}
\hline $\mathbf{5 6 . 8 \%}$ & POS & NEG & P/T & E/P \\
\hline POS(475) & $76.2 \%$ & $23.8 \%$ & 10 & 5.2 \\
\hline NEG(463) & $63.1 \%$ & $36.9 \%$ & 9 & 3.5 \\
\hline \hline $\mathbf{5 1 . 8 \%}$ & ACT & PAS & P/T & A/P \\
\hline ACT(461) & $20.6 \%$ & $79.4 \%$ & 8 & -4.3 \\
\hline PAS(477) & $18.0 \%$ & $82.0 \%$ & 11 & -4.2 \\
\hline
\end{tabular}

Table 5: CM for all Moods

An important set of emotions found in the literature (Ekman, 1972) has been termed the Big Six. These emotions are fear, anger, happiness, sadness, surprise and disgust. We have used a minimally extended set, adding love and desire (Cowie and Cornelius, 2002), to cover all four quadrants (we called this set the Big Eight). Fear, anger and disgust belong to quadrant 1, sadness and surprise (we have taken it to be a synonym of 'taken aback' in the typology) belong to quadrant 2, love and desire (taken to be synonyms of 'amorous' and 'longing' in the typology) belong to quadrant 3 and happy to quadrant 4 . Table 6 shows the results for the classification of the blog posts that were tagged with one of these emotions. This amounts to classifying the posts containing only the Big Eight affective states.

\begin{tabular}{|c|c|c|c|c|}
\hline $\mathbf{5 9 . 0 \%}$ & POS & NEG & P/T & E/P \\
\hline POS(467) & $72.4 \%$ & $27.6 \%$ & 9 & 5.1 \\
\hline NEG(351) & $58.7 \%$ & $41.3 \%$ & 6 & 2.3 \\
\hline \hline $\mathbf{5 4 . 9 \%}$ & ACT & PAS & P/T & A/P \\
\hline ACT(357) & $23.8 \%$ & $76.2 \%$ & 8 & -4.4 \\
\hline PAS(461) & $21.0 \%$ & $79.0 \%$ & 8 & -4.6 \\
\hline
\end{tabular}

Table 6: CM for the Big Eight

In the remaining two experiments, blog posts have been classifed using a discrete scoring system. Disregarding the real value of SO, each pattern was scored with a value of +1 for a positive score and -1 for a negative score. This amounts to counting the number of patterns on each side and has the advantage of providing a normalized value for $\mathrm{E} / \mathrm{T}$ and $\mathrm{A} / \mathrm{T}$ between -1 and +1 . Normalized values are the first step towards a measure of affect, not merely a score, in the sense that it gives an estimate of the strength of affect. We have not 
classified the posts for which the resulting score was zero, which means that even fewer posts (741) than the previous experiment were actually evaluated. Table 7 shows the results for all moods and table 8 for the Big Eight.

\begin{tabular}{|c|c|c|c|c|}
\hline $\mathbf{5 5 . 7 \%}$ & POS & NEG & P/T & E/P \\
\hline POS(374) & $53.2 \%$ & $46.8 \%$ & 11 & 0.03 \\
\hline NEG(367) & $41.7 \%$ & $58.3 \%$ & 9 & -0.11 \\
\hline \hline $\mathbf{5 3 . 3 \%}$ & ACT & PAS & P/T & A/P \\
\hline ACT(357) & $21.8 \%$ & $78.2 \%$ & 8 & -0.3 \\
\hline PAS(384) & $17.4 \%$ & $82.6 \%$ & 12 & -0.34 \\
\hline
\end{tabular}

Table 7: CM for all Moods: Discrete scoring

\begin{tabular}{|c|c|c|c|c|}
\hline $\mathbf{5 9 . 8 \%}$ & POS & NEG & P/T & E/P \\
\hline POS(373) & $52.3 \%$ & $47.7 \%$ & 10 & 0.01 \\
\hline NEG(354) & $32.2 \%$ & $67.8 \%$ & 9 & -0.2 \\
\hline \hline $\mathbf{5 2 . 8 \%}$ & ACT & PAS & P/T & A/P \\
\hline ACT(361) & $25.8 \%$ & $74.2 \%$ & 10 & -0.3 \\
\hline PAS(366) & $20.5 \%$ & $79.5 \%$ & 9 & -0.4 \\
\hline
\end{tabular}

Table 8: CM for the Big Eight: Discrete scoring

\subsection{Analysis of Results}

Our concerns about the paradigm words for evaluating the activity dimension are clearly revealed in the classification results. The classifier shows a heavy negative (passive) bias in all experiments. The overall accuracy for activity is consistently below that for evaluation: three of them are not statistically significant at $1 \%(51.8 \%, 53.3 \%$ and $52.8 \%$ ) and two at even 5\% (51.8\% and 52.8\%). The classifier appears particularly confused in table 5, averaging a score for active posts (-4.3) smaller than for passive posts (-4.2). It is not impossible that the moods present in the typology may have to be shifted towards the passive dimension, but further research should look first at finding better paradigm words for activity. A good starting point for the calibration of the classifier for activity is the creation of a list of humanannotated words for activity, comparable in size to the GI list, combined with an experiment similar to the one for which results are reported in table 3.

With regards to the evaluation dimension, tables 5 and 6 reveal a positive bias (despite having a classifier which has a 'built-in' negative bias, see section 4.1). Possible explanations for this phenomenon include the use of irony by people in negative posts, blogs which are expressed in more positive terms than their annotation would suggest, and failure to detect 'negative' contexts for patterns - one example of the latter is provided in table 9. This phenomena appears to be alleviated

\begin{tabular}{|c|c|}
\hline Mood: & bored (evaluation-) \\
\hline Post: & $\begin{array}{l}\text { gah!! i need new music, any } \\
\text { suggestions? by the way, } \\
\text { GOOD MUSIC. }\end{array}$ \\
\hline Patterns: & $\begin{array}{l}\text { new music }[\mathrm{JJ} \mathrm{NN}]+4.38 \\
\text { GOOD MUSIC }[\mathrm{JJ} \text { NN] }+53.40\end{array}$ \\
\hline Average SO: & +57.78 (evaluation+) \\
\hline
\end{tabular}

Table 9: Missclassified post

by the use of discrete scores (see tables 7 and 8). One way of refining the scoring system is to reduce the effect of scoring antonyms as high as synonyms by not counting co-occurences in the corpus where the word 'not' is in the neighbourhood (Turney, 2001). Also,

The long-term goal of this research is to be able to classify texts by locating their normalized scores for evaluation and activity between -1 and +1 , and we have suggested a simple method of achieving that by averaging over discrete scores. However, by combining individual results for evaluation and activity for each post ${ }^{13}$, we can already classify text into one of the four quadrants, and we can expect the average accuracy of this classification to be approximately the product of the accuracy for each dimension. Table 10 shows the results for the classification directly into quadrants of the 727 posts already classified into halves $(\mathrm{E} \pm, \mathrm{A} \pm)$ in table 8 . The overall accuracy is $31.1 \%$ (expected accuracy is $59.8 \% * 52.8 \%=$ $31.6 \%$ ). There are biases towards Q2 and Q3, but no clear cases of confusion between two or more classes.

\begin{tabular}{|c|c|c|c|c|}
\hline 31.1\% & Q1 & Q2 & Q3 & Q4 \\
\hline Q1(180) & $21.1 \%$ & $47.8 \%$ & $22.2 \%$ & $8.9 \%$ \\
\hline Q2(174) & $15.5 \%$ & $51.1 \%$ & $25.3 \%$ & $8.0 \%$ \\
\hline Q3(192) & $9.9 \%$ & $42.2 \%$ & $40.1 \%$ & $7.8 \%$ \\
\hline Q4(181) & $9.4 \%$ & $33.7 \%$ & $44.8 \%$ & $12.2 \%$ \\
\hline
\end{tabular}

Table 10: CM for Big Eight: Discrete scoring

Finally, our experiments show no correlation between the length of a post (in number of patterns) and the accuracy of the classification.

\footnotetext{
${ }^{13}$ For example, a post with E- and A+ would be classified in Q1.
} 


\section{Conclusion and Future Work}

In this paper, we have used a machine learning approach to show that there is a relation between the semantic content of texts and the affective state they (wish to) convey, so that a typology of affective states based on semantic association is a good description of the distribution of affect in a twodimensional space. Using automated methods to score semantic association, we have demonstrated a method to compute semantic orientation on both dimensions, giving some insights into how to go beyond the customary 'sentiment' analysis. In the classification experiments, accuracies were always above a random baseline, although not always statistically significant. To improve the typology and the accuracies of classifiers based on it, a better calibration of the activity axis is the most pressing task. Our next steps are experiments aiming at refining the translation of scores to normalized measures, so that individual affects can be distinguished within a single quadrant. Other interesting avenues are studies investigating how well the typology can be ported to other textual data domains, the inclusion of a 'neutral' tag, and the treatment of texts with multiple affects.

Finally, the domain of weblog posts is attractive because of the easy access to annotated data, but we have found through our experiments that the content is very noisy, annotation is not always consistent among 'bloggers', and therefore classification is difficult. We should not underestimate the positive effects that cleaner data, consistent tagging and access to bigger corpora would have on the accuracy of the classifier.

\section{Acknowledgement}

This work was partially funded by the European Commission through the Network of Excellence EPOCH ("Excellence in Processing Open Cultural Heritage"). Thanks to Peter Turney for the provision of access to the Waterloo MultiText System.

\section{References}

Eric Brill. 1994. Some advances in transformationbased part of speech tagging. Proc. of 12th National Conference on AI. pp. 722-727. Menlo Park, CA: AAAI Press.

Kenneth Ward Church and Patrick Hanks. 1990. Word association norms, mutual information, and lexicography. Computational Linguistics. Vol. 16, No 1. pages 22-29, MIT Press, Cambridge, MA, USA.
Roddy Cowie and Randolph R. Cornelius. 2002. Describing the emotional states that are expressed in speech. Speech Communication 1228. Elsevier Science B.V.. 20 June 2002, 28 pages.

Paul Ekman. 1972. Universal and cultural differences in facial expression of emotion. J.K. Cole (Eds), Nebraska Symposium on Motivation. pp 207-282. Lincoln, University of Nebraska Press.

Thorsten Joachims. 2001. Learning to Classify Text Using Support Vector Machines. Kluwer Academic Publishers.

Jaap Kamps and Robert J. Mokken and Maarten Marx and Maarten de Rijke. 2004. Using WordNet to measure semantic orientation of adjectives. Proc. of LREC 2004. Vol. IV, pages 1115-1118.

Gilad Mishne. 2005. Experiments with mood classification in blog posts. In Style2005 - the 1st Workshop on Stylistic Analysis Of Text For Information Access, at SIGIR 2005.

Charles E. Osgood, George J. Suci, and Percy H. Tannenbaum. 1957. The Measurement of Meaning. University of Illinois.

Klaus R. Scherer. 1984. Emotion as a Multicomponent Process: A model and some cross-cultural data. In P. Shaver (Ed.) Review of Personality and Social Psych. Vol. 5 (pp. 37-63). Beverley Hills, CA: Sage.

H. Schmid. 1994. Probabilistic part-of-speech tagging using decision trees. In International Conf. on New Methods in Language Processing. Manchester UK.

Marc Schröder and Roddy Cowie. 2005. Towards emotion-sensitive multimodal interfaces. Invitated talk at the Workshop on "Adapting the interaction style to affective factors" pp. 235-253. User Modelling 2005, July 25, Edinburgh.

Philip J. Stone and Dexter C. Dunphy and Marshall S. Smith and Daniel M. Ogilvie. 1966. The General Inquirer: A Computer Approach to Content Analysis. MIT Press. http://www. webuse. umd. edu $: 9090 /$.

Gene F. Summers. 1970. Attitude measurement. Chicago: Rand McNally. pp. 235-253.

Peter Turney. 2001. Mining the Web for Synonyms: PMI-IR versus LSA on TOEFL. European Conference on Machine Learning. pp 491-502. citeseer.nj.nec.com/ turney01mining.html.

Peter D. Turney. 2002. Thumbs Up or Thumbs Down? Semantic Orientation Applied to Unsupervised Classification of Reviews. Proc. of the ACL 2002. Philadelphia, USA. July 8-10, 2002, pp 417 424.

Peter D. Turney and Michael L. Littman. 2003. Measuring praise and criticism: Inference of semantic orientation from association. ACM Trans. Inf. Syst. 21(4):315346. 